\title{
NOTE SUR LA DÉCOUVERTE D'AMPHORES DRESSEL 2/4 ITALIQUES, TARDIVES, À SAINT-ROMAIN-EN-GAL (RHÔNE)
}

\author{
par Armand DESBAT et Hugues SAVAY-GUERRAZ
}

La fouille des entrepôts situés à l'est de la maison des dieux Océans a révélé l'existence de dépôts d'amphores, datables des II $^{\text {e }}$ et III $^{\mathrm{e}} \mathrm{s}$. et constitués pour l'essentiel de Dressel 2/4 dont l'origine italique a été confirmée par des analyses physico-chimiques. Plusieurs groupes de composition sont représentés dont l'un est attribuable à la région du Vésuve. La chronologie de ces dépôts soulève la question de l'arrêt des productions de Dressel 2/4 en Italie et de leur diffusion. Même si l'on admet qu'une partie du matériel est résiduel, ces dépôts constituent un témoin particulièrement intéressant de la diffusion du vin d'Italie en Gaule et notamment du vin du Vésuve après 79 de notre ère.

The digging of the docks at the east of the "Maison des dieux Océans" has revealed the existence of amphora deposits dated from the 2nd and 3rd centuries A. D. They are moslly composed of Dressel 2/4, whose Italic origin has been confirmed by chemical analysis. Several groups of composition have been found, one of them belonging to the Vesuvian area. The chronology of those deposits raises the question of the end of the production of Dressel 2/4 amphora in Italy, and of their exportation. Those deposits nevertheless constitute an evidence of Vesuvian wine in Gaul after 79 A. D.

Le quartier de Saint-Romain-en-Gal, sur la rive droite du Rhône, est caractérisé par la juxtaposition de grandes maisons, d'entrepôts, de locaux artisanaux et de boutiques. En 1986, la fouille a porté sur le bâtiment situé à l'est de la maison des dieux Océans ${ }^{1}$.

Cet ensemble $(73 \mathrm{~m} \times 19 \mathrm{~m})$ comprend au sud un atelier de foulons associé à un habitat (pièces 1 à 10) et, au nord, trois groupes de cellules d'entrepôts desservis par trois couloirs (pièces 11,20 et 27) qui communiquent du côté est avec la rue ${ }^{2}$ (fig. 1).

1 C. Laroche, II. Savay-Gufrraz, Saint-Romain-enGal, Guides archéologiques de la France, 1984. De 1980 à 1985, les fouilles ont porté sur la maison des dieux Océans. L'étude maintenant achevée est en cours de publication.

2 La pièce 16 comporte un vide sanitaire d'amphores Dressel 20 aménagé sous le sol en terre battue.
A l'extrémité nord, dans les pièces 24 à 29 , nous avons pu fouiller une partie des sols du dernier état épargnés par le décapage général du site. C'est ainsi qu'a été découvert un lot important d'amphores composé en majorité de Dressel 2/4, réparties en deux ensembles.

Le premier provient de la pièce 28 qui était encore en partie occupée par une éminence de terrain de $3 \mathrm{~m}$ sur $5 \mathrm{~m}$ et d'une hauteur de $0,60 \mathrm{~m}$. Il s'agissait d'une accumulation de chaux et de tessons d'amphores intimement mêlés. L'ensemble recalcifié constituait un amas fortement concrétionné qui avait été entaillé en plusieurs points par des fosses modernes. Plus de $400 \mathrm{~kg}$ d'amphores ont été recueillis.

Quelques blocs de calcaire non totalement calcinés ont été retrouvés noyés dans la chaux; on identifie un petit chapiteau corinthien à feuilles lisses 

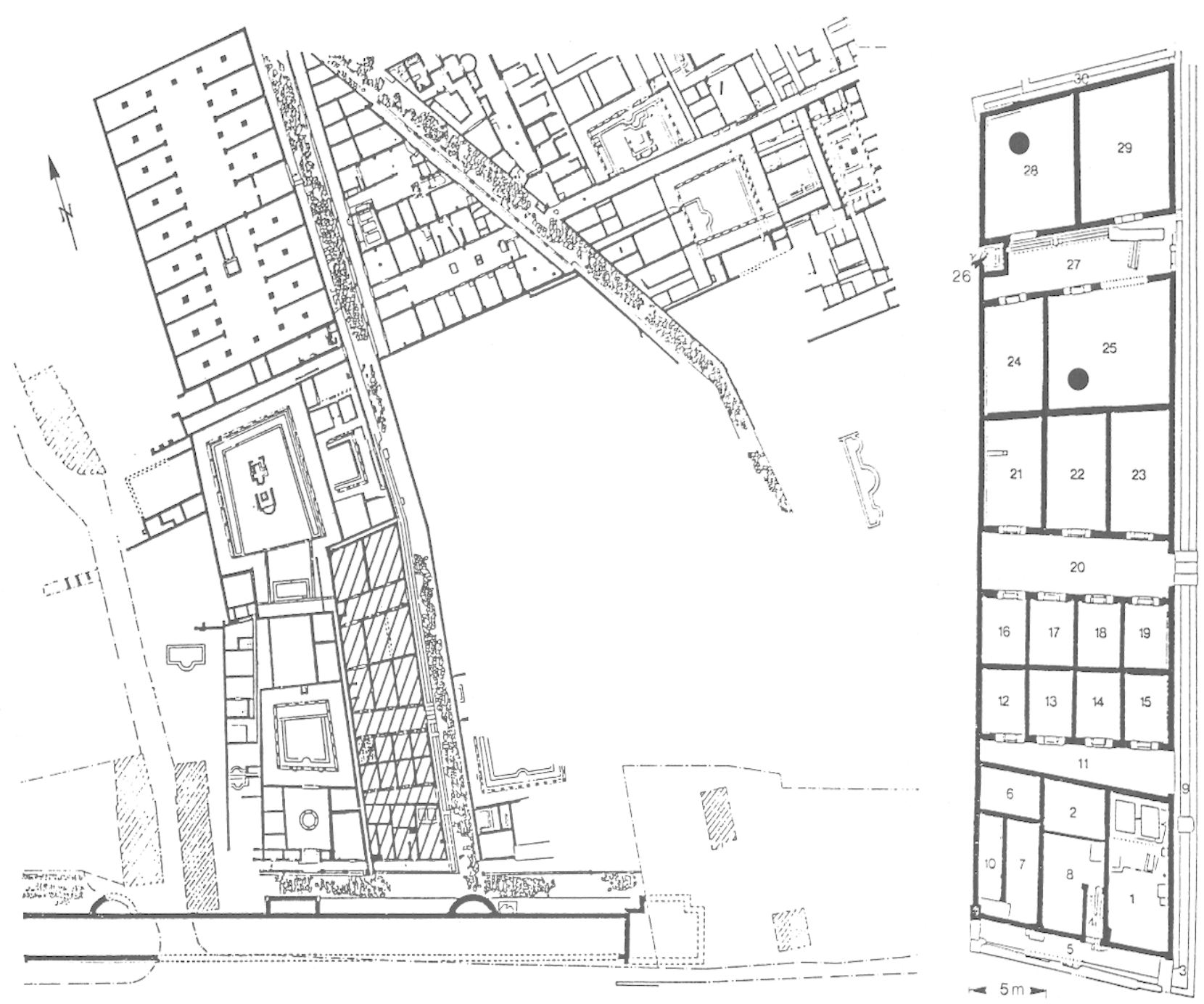

Fig. 1 - A gauche, plan du site avec la situation des entrepôts (en tramé) à l'est de la maison des dieux Océans; à droite, les petits entrepôts avec la localisation des découvertes $(\bullet)$.

et un petit autel mouluré. Enfin, de nombreux clous et une série de huit charnières en bronze identiques proviennent sans doute des planches de récupération brûlées pour la préparation de la chaux.

L'absence de structure de combustion et le fait que les tessons d'amphores ne portent pas de trace de recuisson montrent que l'on n'est pas à l'emplacement même du four à chaux, mais que l'on a le résultat du mélange d'un double apport dont les origines et la fonction nous sont inconnues.

Cet ensemble reposait sur la couche charbonneuse correspondant à l'incendie du plancher qui formait le sol de la pièce dans son état final ${ }^{3}$. Il est donc postérieur à la destruction de l'édifice.

3 Celui-ci était matérialisé par des alignements de blocs, de taille irrégulière, qui calaient les solives et par la trace d'une de ces solives calcinées. Le plancher reposait en outre sur la semelle des murs.
Toutes les données dont nous disposons actuellement conduisent à placer l'abandon du quartier et des entrepôts dans le courant du $\mathrm{II}^{\mathrm{e}} \mathrm{s}$. après J.-C. ${ }^{4}$. La fouille de la pièce elle-même a livré peu d'éléments de datation, mais les couches d'abandon de l'édifice contenaient des fragments de céramique culinaire africaine (Hayes 196 et 197) ainsi qu'une monnaie de Flavius Victor (287-288).

En dehors de quelques amphores Gauloises 4 et 5 , le dépôt était constitué de très nombreux fragments d'amphores Dressel 2/4, dont on peut estimer le nombre à 52 .

4 Cette datation est fournie par les monnaies du $\mathrm{III}^{\mathrm{e}} \mathrm{s}$. recueillies dans les niveaux d'abandon et par la présence de céramiques (africaines en particulier). Quelques très rares monnaies ou tessons plus tardifs laissent supposer une réoccupation sporadique mais aussi des travaux de récupération dont témoigne la découverte d'un four à chaux en 1982. 

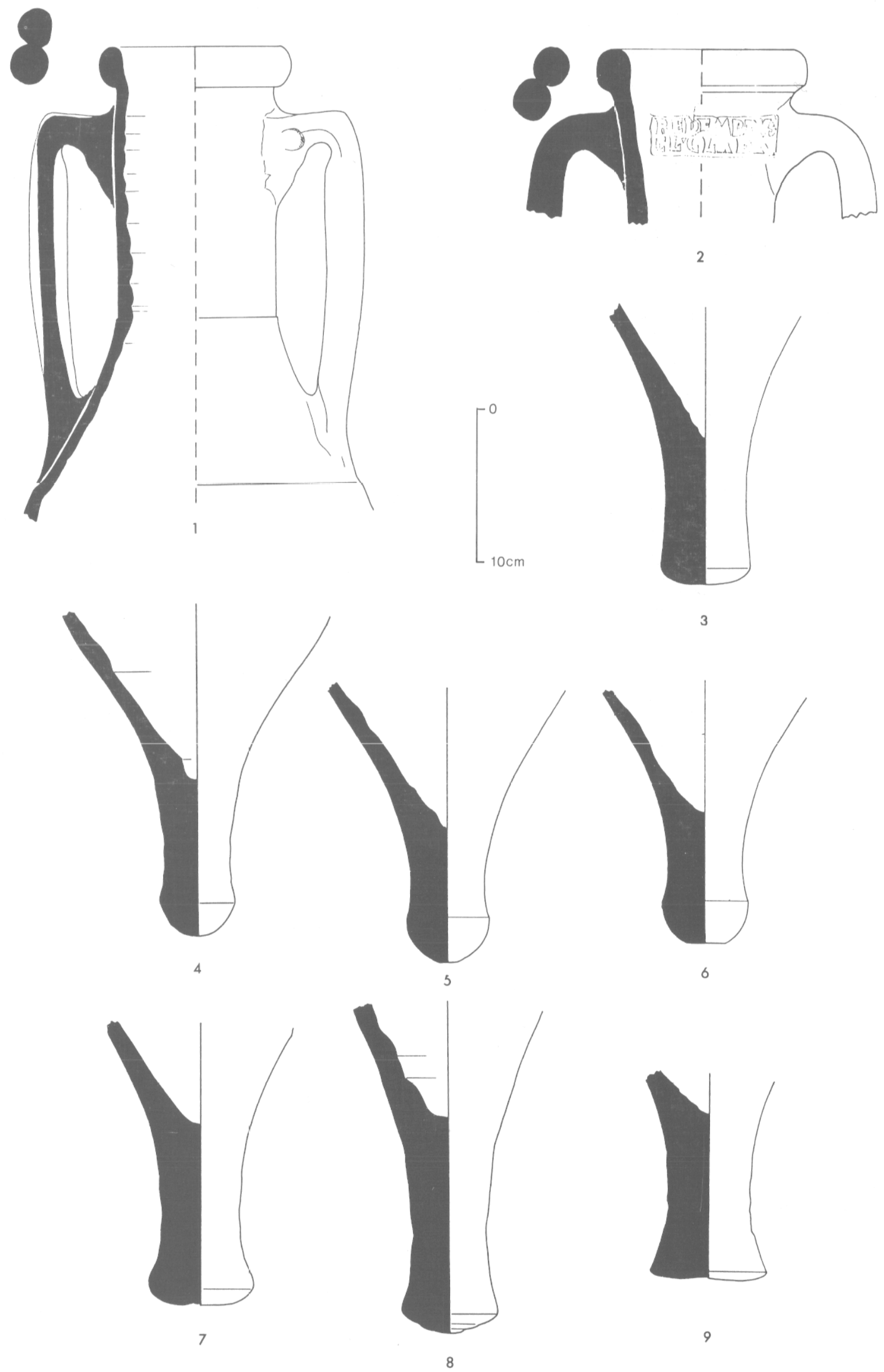

Fig. 2 - Exemples de cols et pilons d'amphores de la pièce 28 . 


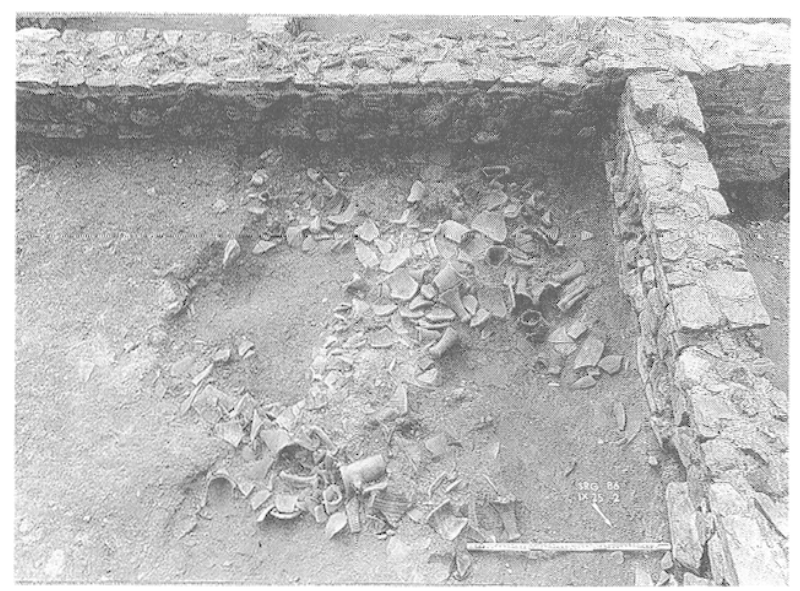

Fig. 3 - Le lot d'amphores de la pièce 25.

Excepté quatre exemplaires, celles-ci se caractérisent par des cols de fort diamètre, des anses larges et épaisses, bifides. Les pilons massifs présentent des formes variées que l'on peut classer en deux groupes: le premier comprend des pilons terminés par une protubérance arrondie à l'extrémité aplatie (fig. ' $2, \mathrm{n}^{\text {os }} 4$ à 6 ) et le second, des pilons plus ou moins évasés et aplatis à leur extrémité (fig. $2, \mathrm{n}^{\text {os }} 7$ à 9 ). Six cols portent des estampilles rectangulaires, disposées pour cinq d'entre elles sur deux lignes:

- REIEMPTVS/CL.CLADI en trois exemplaires (fig. $6, \mathrm{n}^{\circ \mathrm{s}} 1$ à 3 et fig. $2, \mathrm{n}^{\circ} 2$ ),

- une estampille qui a la particularité d'avoir été frappée deux fois (une fois à l'endroit, l'autre à l'envers) ce qui rend sa lecture difficile. On peut lire cependant pour la première ligne ..CIAE et la seconde M.F.VICTRI(CIS) (fig. 6, $\mathrm{n}^{0} 4$ ),

- deux marques incomplètes (fig. $6, \mathrm{n}^{\text {os }} 5$ et 6 ).

Plusieurs fragments montrent en outre des traces de tituli en blanc ou en rouge.

Les pâtes, en majorité de teinte brune ou rouge, présentent toutefois des différences d'aspect assez marquées qui correspondent aux variations morphologiques observées sur les pilons ou les lèvres. Plusieurs amphores présentent des pâtes proches du type Eumachi ${ }^{5}$, riches en minéraux volcaniques, qui permettent de leur attribuer une origine italique confirmée par les analyses (voir appendice, p. 212).

Le second ensemble provient de la pièce 25 située au sud de la première. Il se présentait sous la forme d'un lit d'amphores brisées ennoyé dans une couche riche en éléments de démolition, gravats

5 A. Tchfrnia, F. Zevi, Amphores vinaires de Campanie et de Tarraconaise à Ostie, in: Recherches sur les amphores romaines, home, 197\%, p. 35-67. mêlant débris de mortier de tuileau, fragments de marbre, tesselles de mosaïques (fig. 3). Cette couche occupait toute la surface de la pièce $\left(80 \mathrm{~m}^{2}\right.$ environ) mais n'a été fouillée que sur $9 \mathrm{~m}^{2}$. A l'inverse de l'amas dégagé dans la pièce précédente, cette couche paraît avoir constitué un remblai d'assainissement disposé sous le niveau du plancher dont il semble que la pièce était pourvue. Ce dépôt pourrait, de ce fait, être antérieur à celui de la pièce 29 .

Là encore, la quasi-totalité des fragments recueillis appartiennent à des Dressel 2/4 dont 28 exemplaires ont été décomptés. Leurs caractéristiques sont voisines de celles du premier ensemble sans être toutefois identiques. On observe des variantes typologiques comme dans le premier ensemble. Il apparait un type majoritaire qui associe un col massif, des anses larges (jusqu'à $8 \mathrm{~cm}$ ), bifides, une épaule large très aplatie et un pilon épais terminé par une protubérance hémisphérique (fig. 4, $\left.\mathrm{n}^{\text {os }} 1,2,3,5\right)$. D'autres pilons possèdent une terminaison plus ou moins évasée. Un seul col présentait une estampille incomplète disposée sur deux lignes : ...VNCIONIS/...RP (couronne) (fig. $6, \mathrm{n}^{0} 7$ et 4 , $\mathrm{n}^{\circ} 7$ ). Une particularité est à noter: la résine qui enduisait ces amphores est bien conservée sur de nombreux fragments.

Les pâtes sont pour la plupart riches en éléments volcaniques qui suggèrent une origine italique que les analyses ont confirmée (cf. appendice). On note cependant l'absence des pâtes de type Eumachi présentes dans le premier ensemble.

Les comparaisons typologiques conduisent ègalement à attribuer à l'Italie, en particulier à la Campanie la plupart des amphores des deux ensembles. L'étude réalisée par Panella et $\mathrm{Fano}^{6}$ sur les amphores de Pompéi montre plusieurs exemplaires ayant des caractéristiques proches de celles de nos amphores (fig. 7). Plusieurs présentent notamment un pilon "en boule» dans leur groupe $3^{7}$, tandis que dans leur groupe 4 figurent des amphores massives avec des pilons larges qui se rapprochent du type majoritaire de la pièce $25^{8}$.

Les caractéristiques des anses, des cols et des lèvres sont elles-mêmes tout à fait comparables. Un argument supplémentaire est fourni par les tituli relevés sur les amphores de Pompéi. Il s'agit d'inscriptions peintes en blanc et en rouge comme

6 C. Panella, M. Fano, Le anfore con anse bifide conservate a Pompei, in: Méthodes classiques et méthodes formelles dans l'étude des amphores, Rome, 1977, p. 133-177.

7 Panella, Fano, op. cit., fig. 8, 9, 11, 18, 22, 23 et 24.

8 Panella, Fano, op. cit., fig. 39, 40, 41, 42, 44, 45, 46 et 48 . 


$$
\begin{aligned}
& \text { ఏ } \\
& \left.\prod_{1}\right)
\end{aligned}
$$



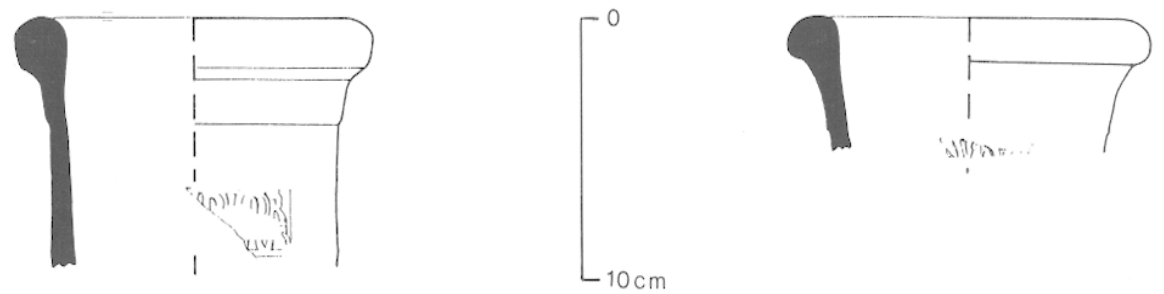

Fig. 5 - Cols estampillés de la pièce 22 .

sur nos exemplaires avec une graphie qui présente une grande similitude. La lecture SVR(rentinum vinum) sur une des Dressel 2/4 de Saint-Romain apporte encore un argument supplémentaire pour attribuer à la Campanie certaines des amphores qui composent nos deux ensembles (fig. 8).

D'autres exemplaires proviennent d'un remblai identique à celui de la pièce 25 , répandu dans la pièce 29 , ainsi que des niveaux supérieurs de la pièce 22 (fig. 5). Ils ont livré trois autres estampilles incomplètes, disposées pour les deux premières sur deux lignes ..VDIOR/...TVS, (M)ARTIAL/... et $\ldots A V D I O(R)$ (fig. $6, \mathrm{n}^{\circ \mathrm{s}} 8$ à 10 ).

Les contextes chronologiques dans lesquels ont été découvertes ces amphores ne peuvent manquer de susciter des interrogations. En effet, les amphores produites dans la région de Pompéi sont rares, pour ne pas dire exceptionnelles, après l'éruption de 79 . Dc plus, il est admis de longue date que les amphores du type Dressel 2/4 ont disparu en Italie après $150^{\circ}$. Les quantités recueillies à Saint-Romain et leur état de conservation semblent contredire l'hypothèse d'un matériel résiduel ${ }^{10}$. S'il s'agissait d'amphores du ${ }^{\text {er }}$ s., il faudrait admettre que l'on ait stocké pendant plus d'un siècle des amphores entières pour les briser à la fin du $\mathrm{II}^{\mathrm{e}} \mathrm{s}$. et au début du $\mathrm{Ir}^{\mathrm{e}} \mathrm{s}$. Bien que peu probable, cette hypothèse n'en est pas pour autant impossible mais d'autres indices tendent à démontrer qu'il s'agit bien d'amphores italiques tardives. Malgré leurs ressemblances avec les amphores de Pompéi, les amphores de Saint-Romain se distinguent de ces dernières par la présence de timbres sur

9 Le point le plus récent sur cette question est donné par A. Tchernia, Le vin de l'Italie romaine, Bibliothèque des Écoles Françaises d'Athènes et de Rome, 260, 1986, chap. V. Voir aussi A. TChernia, Quelques remarques sur le commerce du vin et des amphores, in : The Seaborne Commerce of Ancient Rome : Studies in Archaeology and Hislory, MAAR, 36, p. 305312 .

10 C'est le cas pour les nombreux tessons de Dressel 1 qui subsistent dans les remblais de 15-20 après $\mathrm{J}$.-C. à SaintRomain, mais on peut constater qu'il s'agit de tessons usés. le col qui sont absents sur les amphores pompéiennes $d u \quad I^{\text {er }} \mathbf{s}$. Il faut noter surtout la rareté dans les contextes du $I^{\text {er }} \mathrm{s}$., à Saint-Romain-en-Gal comme à Lyon, des amphores Dressel $2 / 4$ italiques ${ }^{11}$. A l'inverse, les contextes des $\mathrm{II}^{\mathrm{e}}$ et $\mathrm{III}^{\mathrm{e}} \mathrm{s}$. ont livré fréquemment des fragments d'amphores Dressel 2/4 italiques généralement considérés comme résiduels, mais dont on peut se demander, après la découverte de Saint-Romain, si tel est bien le cas. Elles présentent souvent des caractéristiques qui les rapprochent de nos exemplaires (grosseur des anses, diamètre des cols) et les distinguent de la plupart des exemplaires $d u$ I $^{\text {er }} \mathrm{s}$. Bien que peu nombreuses, ces amphores arrivent à représenter un pourcentage quelquefois plus élevé qu'à la fin du $\mathrm{I}^{\mathrm{er}} \mathrm{s}$. (ainsi, dans un dépotoir de la rue des Farges du début du $111^{2}$ s., les Dressel 2/4 italiques comptent pour $6 \%$ des amphores vinaires sur un lot de 120 amphores, contre $2 \%$ dans le dépotoir flavien du Bas-deLoyasse sur un lot de 427 amphores).

Par ailleurs, les données récentes fournies par Cl. Panella sur des contextes de la fin du ${ } \mathrm{I}^{\mathrm{e}} \mathrm{s}$. en Italie, notamment à Ostie, tendent à démontrer que l'amphore Dressel 2/4 n'a pas disparu après 150 : malgré une baisse sensible des pourcentages, les Dressel $2 / 4$ italiques représentent encore $4,05 \%$ à Ostie dans les contextes de 160-180, 3,66\% dans ceux datés de 190-210 et comptent pour 17,95\% dans les contextes tardo-antonins de Settefinestre ${ }^{12}$.

Il faut signaler encore quelques découvertes de Dressel 2/4 avec les estampilles sur le col comparables aux nôtres, dans des contextes des $1 I^{e}$ et $11 I^{e} \mathrm{~s}$. Deux de ces découvertes se situent en Orient, la première à Corinthe, où l'on retrouve la même estampille que celle provenant de la pièce 28 : CAEDICAE/M.F.VICTRICIS dans un contexte du

11 Dans l'horizon SRG 3 (15-20 après J.-C.), les Dressel $2 / 4$ italiques comptent pour $8,5 \%$ des amphores vinaires, dans SRG 4 (40-60 après J.-C.) pour 9,5\%, mais dans le dépotoir flavien du Bas-de-Loyasse à Lyon pour $2 \%$ seulement.

12 Cf. C. Panella, Le anfore italiche del II secolo D. C., in: Amphores romaines et histoire économique. Dix ans de recherches, École Française de Rome, 114, 1989, p. 139-178. 

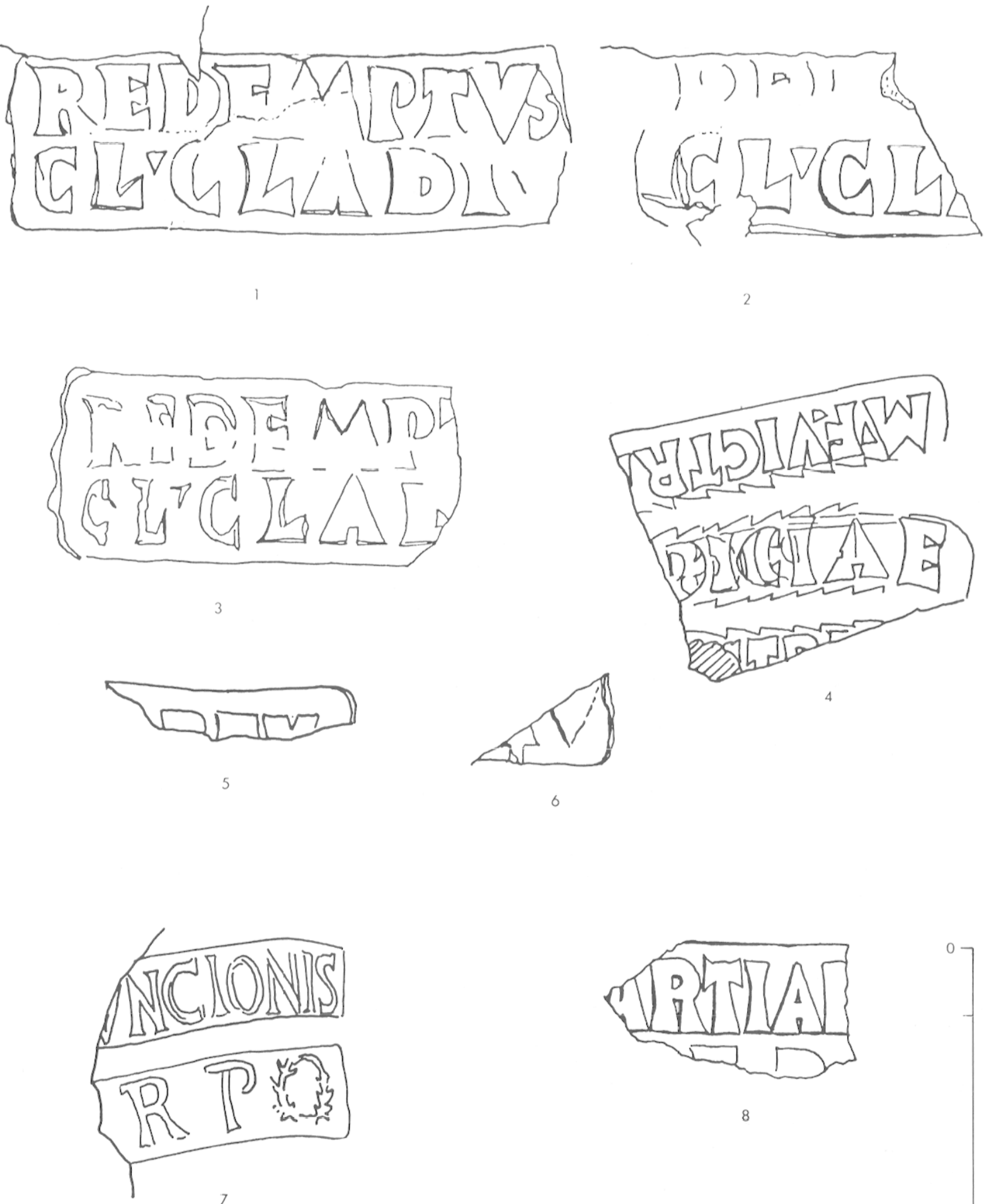

8
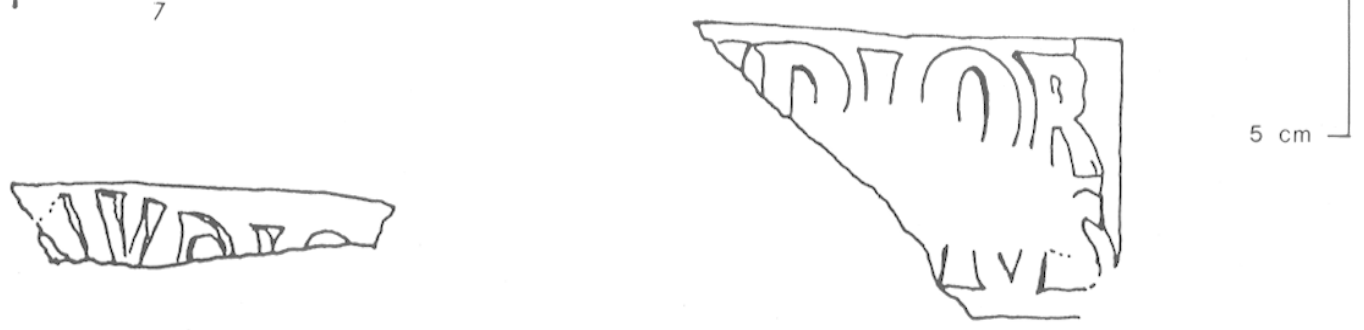

10

Fig. 6 - Estampilles sur les cols d'amphores. 


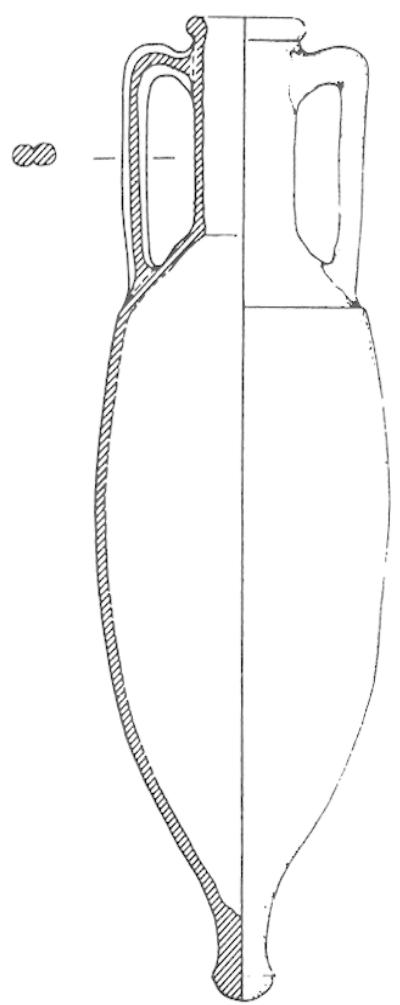

- Gruppo 3. Pompei, n. 91.

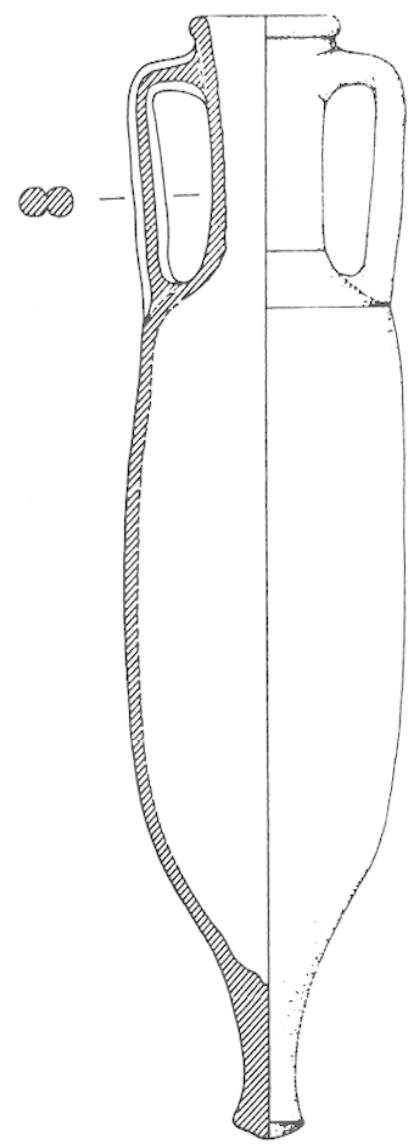

- Gruppo 8. Pompei, n. 469.

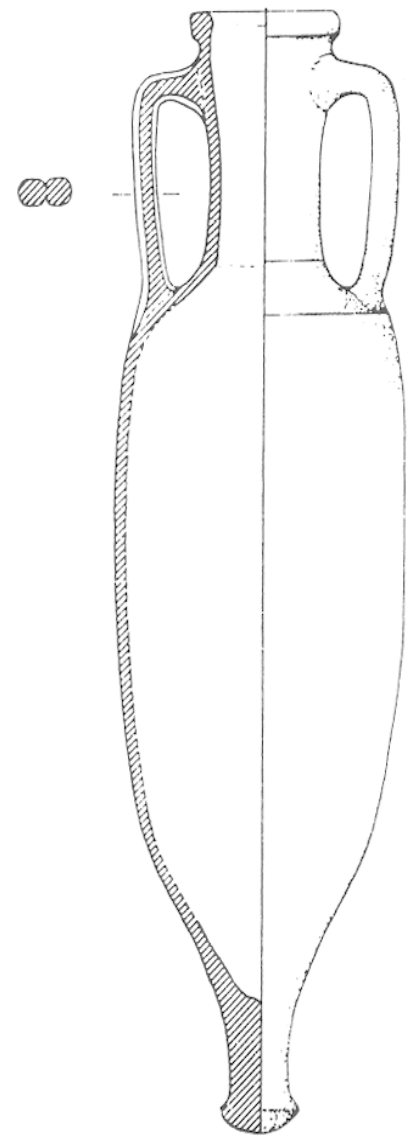

Gruppo 8. Pompei, n. inv. 14190.

Fig. 7 - Type d'amphores de Pompéi (d'après Panella, Fano, 1977).

III $\mathrm{S}^{\mathrm{e}}{ }^{13}$ et la seconde à Salamine de Chypre où deux cols d'amphores ont livré les estampilles CORNELLI POLLIO/SILVANVS $F$ et $M A R / / /^{14}$. Plus intéressan-

13 C. K. Williams, O. H. Zrrvos, Corinth 1984 : East of the Theater, Ilesperia, 1985, p. 56 et 57 , pl. 8, no 1 . La marque CAEDICAE/M.F.VICTRICIS est signalée d'autre part dans II. Callender, Roman Amphorae with Index of Stamps, Oxford, 1965, p. 86, no 218, à Rome et Florence associèe à la marque MARTINALIS/SER dans un cartouche différent et sous la forme CAEDICAE.M.F.VICTRICIS/ $I O L \ldots$ à Terracine et Carthage. On notera que l'estampille MARTIALIS est à rapprocher de celle trouvée à SaintRomain (fig. 6) (M)ARTIAL et des exemplaires incomplets de Salamine de Chypre MAR ... (cf. note 14 infra). S. MartinKilcher a eu la gentillesse de nous signaler également une Dressel 2/4 italique dans un contexte du $\mathrm{III}^{\mathrm{e}} \mathrm{s}$. à Augst. A Saint-Romain, il faut signaler que les Dressel 2/4 sont abondantes dans le fonds ancien provenant des décapages du site, notamment dans le secteur du "marché".

14 Y. Calvet, Salamine de Chypre. 3, Les timbres amphoriques, Paris, 1972, nos 111, fig. 121 et 112, fig. 122 . Aucune datation n'est proposée pour ces marques mais leur découverte en association avec des lampes à huile à bec rond type Deneauve VIIIB suggère le $\mathrm{II}^{\mathrm{e}}$ ou $\mathrm{III}^{\mathrm{e}} \mathrm{s}$. te encore est la trouvaille effectuée en Italie en 1985, de neuf Dressel 2/4 dans un dépôt datable du début du ${ } I^{\mathrm{e}}{ }^{\mathrm{s}} .^{15}$. Trois estampilles relevées dans ce dépôt et une quatrième trouvée en 1981 fournissent deux exemplaires de la marque CORNELI POLLI(O)/ (SIL)VANVS.F, identique à celle de Salamine, et deux autres de la marque AMPLIATVS/CL.CLADI. Cette dernière nous livre le même nom que les trois estampilles de Saint-Romain, associé cette fois à celui de l'esclave ou de l'affranchi Ampliatus au lieu de Redemptus. Pour l'auteur, J. Freed, l'inscription CL.CLADI désignerait T. Claudius Claudianus, consul suffect en 199 ou 200. Quant à la marque CORNELI POLLIO, elle désignerait C. Iavolenus Calvinus Geminius Capito Cornelius Pollio Squilla Q. Vulcacius Scuppidius Verrus, consul sous Hadrien

15 J. Freed, Late Stamped Dressel 2/4 Amphorae from a Deposit Dated post $200 \mathrm{~A}$. D. at Villa Site 10 on the Via Gabina, in : Amphores romaines et histoire économique. Dix ans de recherches, op. cit., p. 616-617. 

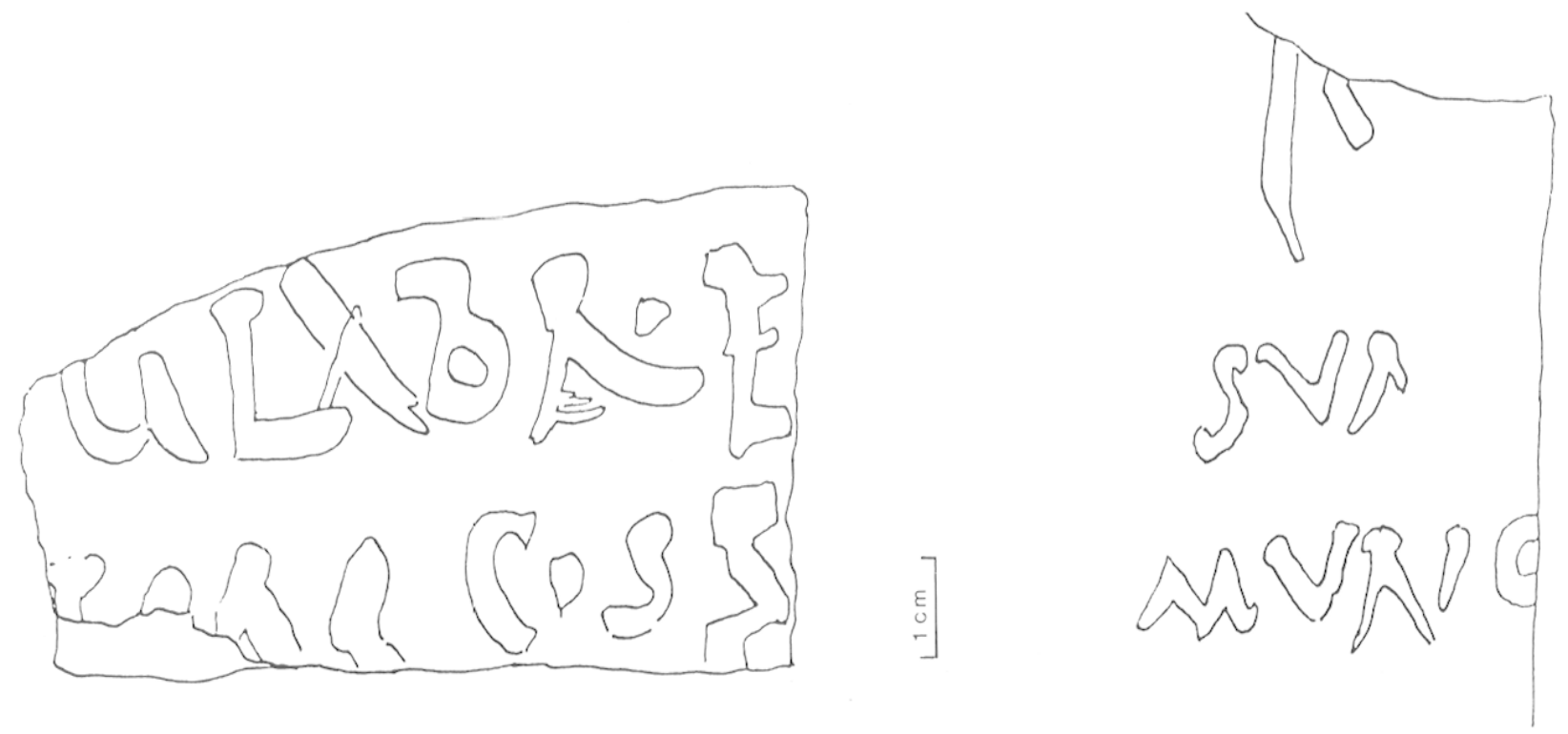

Fig. 8 - Tituli sur les cols du groupe C.

A gauche, sur le premier apparaît une date consulaire avec les noms Glabr(io) et Torq(uato), consuls en 124; à droite, sur le second se lit sur(rentinum vinum).

ou Antonin ${ }^{16}$. Même si l'identification de Cl. Claudianus avec le personnage dont le nom apparaît sur ces amphores peut prêter à discussion, ces découvertes constituent des témoins précieux qui confortent notre propre hypothèse quant à l'origine et à la datation de ces amphores.

La découverte en association avec ces amphores de Gauloises 5, type fréquent sous les Flaviens et au début $\mathrm{du} \mathrm{II}^{\mathrm{e}} \mathrm{s} .{ }^{17}$, oblige à ne pas écarter l'hypothèse d'un matériel résiduel. Un argument en ce sens est donné par la lecture d'une date consulaire sur un fragment issu de la pièce 28 (fig. 8 ) : B. Liou, à qui nous devons la lecture de ce titulus, identifie les consuls cités comme étant Glabrio et Torquatus, consuls en 124 après $\mathrm{J}$.-C.

Nous savons cependant, grâce au témoignage de Galien (XIV, 15), que des vins de Sorrente n'arrivaient à maturité qu'après une vingtaine d'années de conservation et il est donc possible que cette amphore n'ait été commercialisée que bien après sa fabrication.

Le lot d'amphores de Saint-Romain-en-Gal pourrait donc être attribué au plus tôt au premier

16 "Among persons of senatorial rank, the combination of the gentilicium Cornelius and the cognomen Pollio is attested only in the name of the polyonymous $\mathrm{C}$. Iavolenus Calvinus Geminius Capito Cornelius Pollio Squilla Q. Vulcacius Scuppidius Verrus (PIR 2 I 13)", (J. Freed, op. cit., p. 616).

17 F. Laubenheimer, La production des amphores en Gaule Narbonnaise sous le Haut-Empire, Annales litteraires de l'Universilé de Besançon, 66, 1985. quart du II $\mathrm{s}$. Il n'est cependant pas exclu que ce lot soit plus tardif compte tenu des éléments déjà évoqués. Quoi qu'il en soit, il constitue un témoignage de l'importation de vin italique et notamment de Sorrente, en Gaule, au II $^{e}$ s. et fournit l'un des rares ensembles d'amphores campaniennes postérieures à l'ćruption du Vésuve, ce qui lui confère un intérêt certain. Ces amphores ne constituent pas le seul témoin d'importations italiques au II $^{e}$ ou III' $^{e} \mathrm{~s}$. : il faut rappeler en effet que les niveaux de cette époque livrent fréquemment des céramiques à glaçure plombifère d'origine italique et probablement campanienne ${ }^{18}$. Un dépôt du $\mathrm{III}^{\mathrm{e}} \mathrm{s}$. a livré par ailleurs un grand mortier italique dont l'estampille sur deux lignes n'est pas sans rappeler celles relevées sur nos amphores. La découverte de ce dépôt et les parallèles signalés obligent à reconsidérer la date de disparition des Dressel 2/4, qui parait moins assurée qu'il n'y paraissait jusqu'ici. Enfin, l'abondance de ces amphores dans le secteur considéré permet de voir dans ces entrepôts ceux d'un de ces négociants en vins dont on sait par les inscriptions qu'ils constituaient la plus importante corporation de la région lyonnaise.

\section{Armand Desbat et Hugues Savay-Guerraz}

18 A. Desbat, Céramiques à glaçure plombifère des fouilles de Lyon, Figlina, 7, 1986, p. 105-124; - M. Picon et A. Desbat, Note sur l'origine des céramiques à glaçure plombifère, généralement bicolore, des $11^{\mathrm{e}}$ et ${ }^{111^{\mathrm{e}}}$ siècles, de Vienne et Saint-Romain-en-Gal, Figlina, 7, 1986, p. 125-1'27. 


\title{
APPENDICE : ÉTUDE EN LABORATOIRE
}

\author{
par M. Picon et A. Desbat
}

L'étude préliminaire en laboratoire d'une partie de ce matériel a été effectuée, afin, d'une part, de vérifier les données typologiques, lesquelles suggèrent pour ces amphores diverses origines en Italie et, d'autre part, de rechercher des indications éventuelles sur leur localisation, étant entendu qu'une véritable recherche d'origine exigerail un lravail plus ample.

Les analyses ont été faites par fluorescence $\mathrm{X}$, les vingt constituants suivants étant mesurés sur chaque exemplaire : $\mathrm{Na}, \mathrm{K}, \mathrm{Rb}, \mathrm{Mg}, \mathrm{Ca}, \mathrm{Sr}, \mathrm{Ba}, \mathrm{Mn}$, $\mathrm{Ni}, \mathrm{Zn}, \mathrm{Al}, \mathrm{Cr}, \mathrm{Fe}, \mathrm{Si}, \mathrm{Ti}, \mathrm{Zr}, \mathrm{La}, \mathrm{Ce}, \mathrm{P}$ et V. La classification, résumée par le diagramme de la figure 9, se fonde sur les concentrations de huit constituants principaux : K, Mg, Ca, Mn, Al, Fe, Si et Ti. La méthode utilisée est l'analyse de grappes, en affinité moyenne non pondérée, sur variables centrées réduites relatives aux huit constituants précédents.

On observe sur le diagramme de la figure 9 la présence de cinq groupes de composition (marqués de $\mathrm{A}$ à $\mathrm{E}$ ) et de deux exemplaires isolés (cette répartition restant d'ailleurs inchangée si l'on fait intervenir les éléments en traces).

A chacun des groupes de composition et des exemplaires isolés, semble correspondre un type de pied particulier, reporté sur le diagramme de la figure 9, ce qui confirmerait qu'on a bien affaire à des productions issues d'ateliers différents. La seule exception concernerait ici le premier exemplaire isolé en partant de la gauche dont le pied rappelle celui du groupe E. Cette observation n'a rien de surprenant car il semble évident que les caractéristiques typologiques des amphores évoluent en général bien moins vite, d'un atelier à un autre, que les compositions des argiles.

On notera par ailleurs que les six exemplaires estampillés qui ont été analysés se retrouvent tous dans le groupe D et que les amphores du groupe A se distinguent par leur caractère massif et leur grande taille. De plus, on peut remarquer qu'en dehors d'un col estampillé de la pièce 25 qui se regroupe avec ceux de la pièce 28 dans le groupe D, les autres groupes de composition ne se rencontrent que dans l'un ou l'autre ensemble : les groupes A et E dans la pièce 25 , les groupes $\mathrm{B}, \mathrm{C}, \mathrm{D}$ et les deux pilons isolés dans la pièce 28 .

Les caractéristiques visuelles les plus marquantes des pâtes coïncident également avec les groupes de composition : pâtes calcaires à minéraux noirs abondants du groupe $A$, pâtes riches en sanidine et très pauvres en quartz détritique du groupe $B$, pâtes rouges à minéraux noirs abondants du groupe $\mathrm{C}$, pâtes fortement sableuses du groupe $\mathrm{D}$, pâtes très calcaires du groupe E...

La diversité des origines de ces amphores ne fait donc aucun doute mais il est difficile de les préciser : d'une part, à cause de la faiblesse de l'échantillonnage et de l'absence de probabilités a priori en faveur de telle ou telle origine et d'autre part, à cause de l'insuffisance des références d'ateliers. En effet, on connaît selon toute vraisemblance moins d'un atelier d'amphores sur dix en Italie ${ }^{\mathbf{1 9}}$. C'est dire qu'on se contentera de suggérer quelques orientations de recherche, ce qu'il ne faut en aucun cas confondre avec des déterminations d'origine.

Pour le groupe $\mathrm{G}$, c'est vers la région du Vésuve qu'il conviendrait d'orienter les recherches. On connaît en effet six groupes d'amphores Dressel 1 qui possèdent, comme le groupe $\mathrm{C}$, une pâte rouge à minéraux noirs abondants, dont un groupe pompéien ${ }^{20}$. Or, c'est précisément avec ce groupe que les compositions du groupe $\mathrm{C}$ présentent les plus fortes ressemblances. De plus, on sait que ces caractéristiques de composition sont largement répandues dans la région du Vésuve, notamment parmi les tuiles et les céramiques communes. Enfin, on rappelle l'existence pour ces amphores des arguments typologiques qui ont été exposés précédemment en faveur de leur origine vésuvienne.

19 Cf. A. Hesnard, M. Rice, P. Arthur, M. Picon, A. Tchernia, Aires de production des gréco-italiques et des Dr. 1, in: Amphores romaines et histoire économique. Dix ans de recherches, École Française de Rome, 114, 1989, p. 21-65.

20 A. Hesnard el alii, op. cit. 


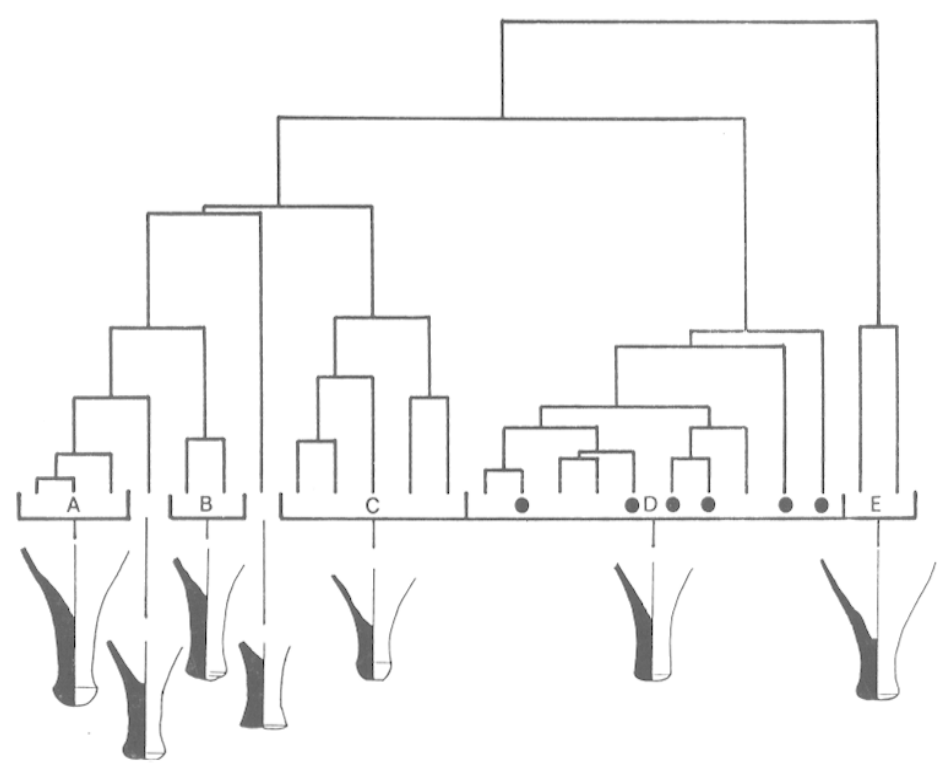

Fig. 9 - Diagramme de la classification par analyse de grappes de 24 exemplaires d'amphores italiques tardives

de Saint-Romain-en-Gal, avec indication des groupes de composition et des formes correspondantes de pieds. Les amphores estampillées sont signalées par un cercle noir.
Pour le groupe B, ce sont les ateliers du Falerne, plus précisément les ateliers situés à l'intérieur des terres au pied du Massique, qu'il faudrait examiner en priorité. Les exemplaires du groupe B présentent en effet des ressemblances de composition avec l'ensemble des six ateliers d'amphores que l'on connaît dans cette région et des similitudes pétrographiques avec les productions de quelques-uns d'entre eux qui se signalent par l'abondance des sanidines et par une quasi-absence de quartz détritique. Bien que ces caractéristiques se rencontrent en d'autres régions, elles méritent d'être signalées car les amphores de Saint-Romain-en-Gal étudiées ici sont des amphores tardives; or, les ateliers du Falerne qui sont à l'intérieur des terres ont fonctionné à une période tardive et au moins jusqu'au premier quart du ${ }{ }^{1 I}{ }^{e}{ }^{2} .{ }^{21}$.

Pour le groupe D, on peut noter des affinités de composition avec les productions du Cécube, plus particulièrement avec celles de l'atelier de Torre San Anastasia : ainsi, leur pâte très sableuse, caractéristique à vrai dire assez banale mais que l'atelier de San Anastasia est le seul des cinq ateliers de la plaine

21 A. Hesnard et alii, op. cit. de Fondi à présenter. Ces indications, manifestement insuffisantes pour une localisation, doivent, plus encore que précédemment, être prises comme une première hypothèse de travail.

Pour le groupe A, enfin, on peut noter que c'est, après le groupe $\mathrm{C}$, celui qui est le moins éloigné des compositions pompéiennes. Les données typologiques exposées précédemment sembient aller dans le même sens.

Quant au groupe E et aux exemplaires isolés, ils ne peuvent actuellement être rapprochés d'aucune région particulière. Il s'agit cependant de productions italiques dont ils présentent certaines caractéristiques pétrographiques et géochimiques parmi les plus fréquentes.

On peut souligner enfin l'intérêt qu'il y aurait à poursuivre les recherches en vue de l'identification de ces différentes amphores; si les premières indications données ici se révélaient exactes, ce serait plus des trois-quarts d'entre elles qui proviendraient de régions d'Italie qui furent à une époque ou à une autre celles de crus particulièrement célèbres, ce qui pourrait expliquer la persistance de ces importations.

M. P. et A. D. 